Rev. Int. Contam. Ambie. 36 (4) 825-834, 2020

https://doi.org/10.20937/RICA.53523

\title{
PREDICCIÓN DE DRENAJE ÁCIDO DE MINA, JALES DE LA MINA LA PRIETA, CHIHUAHUA, MÉXICO
}

Prediction of acid mine drainage, tailings from La Prieta mine, Chihuahua, Mexico

\author{
Fernando Benigno SALAS URVIOLA ${ }^{1,2} *$, Pedro GUADARRAMA GUZMÁN ${ }^{2}$, \\ Georgina FERNÁNDEZ VILLAGÓMEZ ${ }^{2}$, Jesús Fidel GONZÁLEZ SÁNCHEZ ${ }^{2}$ \\ y Luis Adrián BARRAZA TORRES ${ }^{2}$
}

\footnotetext{
${ }^{1}$ Facultad de Ingeniería de Minas, Universidad Nacional del Altiplano Puno, P.O. Box 291, Puno, Perú.

${ }^{2}$ División de Ingenierías Civil y Geomática, Facultad de Ingeniería, Universidad Nacional Autónoma de México, Av. Universidad 3000, Ciudad Universitaria, 04510 Ciudad de México, México.
}

*Autor para correspondencia: bensalas7@hotmail.com

(Recibido: enero de 2019; aceptado: marzo 2020)

Palabras clave: drenaje ácido de mina, jales mineros, predicción.

\begin{abstract}
RESUMEN
La mina La Prieta, Chihuahua, México, fue explotada ampliamente (plata, plomo y cobre) desde la época del virreinato hasta su cierre; por consiguiente, se acumularon grandes cantidades de jales o relaves, producto del procesamiento metalúrgico. Estos jales pueden producir drenaje ácido de mina debido a las reacciones de oxidación e hidrólisis. Con el objetivo de predecir si los jales son generadores de drenaje ácido, se realizó la caracterización de las muestras de jales y la determinación de la posibilidad de generar acidez. Los valores de $\mathrm{pH}$ hallados fueron ligeramente alcalinos (7.8-8.4). Por otro lado, los sulfatos y el azufre total se determinaron mediante los métodos turbidimétrico e infrarrojo, respectivamente. Cabe mencionar que la concentración de azufre total estuvo por debajo del límite de detección del equipo Leco S-144DR y las concentraciones de azufre en forma de sulfatos fueron mínimas. La caracterización mineralógica mostró que sólo en tres muestras se encontró pirita, y en bajos porcentajes. Los minerales calcita y dolomita presentes en el yacimiento tipo skarn intervienen en la neutralización del drenaje ácido de la mina y disminuyen la movilidad de la mayoría de los metales y metaloides tóxicos.
\end{abstract}

Key words: acid mine drainage, mine tailings, prediction.

\begin{abstract}
The La Prieta mine, Chihuahua, Mexico, was intensively exploited (silver, lead and copper) from colonial times until its closure; consequently, large quantities of tailings produced by metallurgical processing accumulated. These tailings can produce acid mine drainage due to oxidation and hydrolysis reactions. With the objective of predicting if tailings can generate acid drainage, tailings samples were characterized and the possibility of generating acidity was investigated. The $\mathrm{pH}$ values found were slightly alkaline (7.88.4). On the other hand, sulfates and total sulfur were determined by turbidimetric and
\end{abstract}


infrared methods, respectively. It is worth mentioning that total sulfur was below the limit of detection of the Leco S-144DR equipment and sulfur concentrations in the form of sulfates were very low. The mineralogical characterization showed that pyrite was found in only three samples and in low percentages. The calcite and dolomite minerals present in the skarn type deposit were involved in the neutralization of acid mine drainage and the decreased mobility of most toxic metals and metalloids.

\section{INTRODUCCIÓN}

México es un país con gran tradición minera, ya que pueblos como los aztecas, los mayas, los zapotecos y los tarascos desarrollaron la minería y la metalurgia en pequeña escala. Hay evidencias de que trabajaron minerales metálicos y no metálicos (SE 2020).

La mina La Prieta dio origen a la ciudad de Hidalgo de Parral, en Chihuahua, México. El primer descubrimiento de plata en ese sitio ocurrió en 1663 y para 1665 operaban más de 60 pequeñas minas. En los últimos años del siglo XIX, Parral vivió un auge minero propiciado por la renovación de los procesos de extracción de plata en la antigua mina del mismo nombre. El año de esplendor de esta mina fue 1926, cuando se extraían 1500 toneladas de minerales puros al día. En 1974 cerró sus puertas y actualmente se encuentra fuera de operación (CEHM 2014).

La explotación de recursos minerales que se encuentran a profundidad requiere el acceso a través de una red de galerías de minería subterránea; en cambio, los que se localizan a poca profundidad se obtienen por minería superficial, cuyo procesamiento genera grandes cantidades de residuos, relaves o jales que ocupan grandes áreas superficiales expuestas al ambiente (Edraki et al. 2014, Nordstrom et al. 2015). La industria minera ha producido y continuará produciendo grandes cantidades de desechos mineros (jales o relaves). Desde la Edad de Piedra se han extraído aproximadamente 1150 millones de toneladas de metales pesados (cobre, plomo, hierro, zinc, cadmio y cromo) con una producción real estimada de relaves mineros que fluctúa entre 5 y 7 mil millones de toneladas anuales en todo el mundo (Edraki et al. 2014).

La eliminación de residuos y relaves mineros tratados y no tratados representa una amenaza y un riesgo significativo de contaminación por acidez con alto contenido de sulfatos y metales pesados en aguas subterráneas y superficiales, humedales, suelo, la cadena alimentaria y los animales (Anawar 2013, Lindsay et al. 2015, Chopard et al. 2017, Lèbre et al. 2017, Plaza et al. 2018). Las partículas finas de relaves en suspensión desempeñan un papel importante en el transporte de metales potencialmente tóxicos. Las personas pueden verse expuestas a este tipo de partículas a través del suelo, el agua y la biota contaminados con este tipo de residuos (Khademi et al. 2018).

El drenaje ácido de mina (DAM) es uno de los desafíos ambientales más importantes que enfrenta la industria minera en todo el mundo, debido a que el agua que se infiltra a través de los minerales de sulfuro metálico tiene valores bajos de $\mathrm{pH}$, con alto contenido de metales y metaloides. Los residuos de las plantas de procesamiento de minerales y las filtraciones de presas de relaves se vuelven ácidos, y esta solución permite que los metales se transporten en sus formas solubles, lo cual puede alterar la calidad del agua, el suelo y la biota (Sheoran y Sheoran 2006, Sánchez-Andrea 2014, Johnson y Hallberg 2005, Candeias et al. 2014, Pabst et al. 2018, Yang et al. 2019). Muchos de los métodos utilizados para remediar el DAM tienen una implementación limitada debido a su bajo rendimiento, imprecisiones de diseño, dificultad para comprender su funcionalidad, altos costos, uso de sustancias químicas peligrosas, agotamiento de los recursos naturales y generación de más desechos (Moodley et al.2018).

En ciertos jales que contienen minerales alcalinos, el $\mathrm{pH}$ suele ser ligeramente alcalino, lo cual indica la presencia de carbonatos reactivos (Zhou et al. 2017). Éstos se enriquecen con una cantidad variable de sulfuros y carbonatos minerales que generan un grado variable de contaminación controlada por el proceso de atenuación natural (Anawar 2013). Estos compuestos, formados por sulfuros primarios y calcita, se corresponden probablemete con los desechos producidos por el procesamiento del mineral proveniente esencialmente del tipo de yacimiento skarn y los minerales secundarios extraídos de chimeneas y mantos

La presencia de calcita y dolomitas influye en la especiación, neutraliza el DAM y disminuye la movilidad de la mayoría de los metales tóxicos y metaloides. La ocurrencia de calcita y dolomitas en este tipo de yacimientos promueve la retención de $\mathrm{As}, \mathrm{Ba}, \mathrm{Cd}$ y $\mathrm{Pb}$, y aumenta la liberación de $\mathrm{Sb}$ (Armienta et al. 2012). 
Çelebi y Öncel (2016) concluyeron en su investigación que en las pruebas estáticas de las muestras de relaves, obtenidas en 10 puntos diferentes, los valores de potencial neto de producción de ácido (PN/PA) fueron positivos. Asimismo, la generación neta de ácido (GNA) estuvo por encima del $\mathrm{pH}$ de referencia de 4.5, por lo tanto no estaba claro si las muestras de relaves podrían formar drenaje ácido. Por lo anterior estos autores aplicaron el procedimiento de lixiviación de las muestras con agua. El pH obtenido de los lixiviados fue de 7.0, lo cual puede explicarse por la presencia de roca caliza, que neutraliza la acidez. También realizaron pruebas cinéticas, las cuales tampoco mostraron ningún efecto al contenido de humedad y a la edad de los relaves en la formación de drenaje ácido.

La investigación realizada por Armienta et al. (2012) en Zimapán, México central, indica que la presencia de calcita interviene en la neutralización del DAM y disminuye la movilidad de la mayoría de los metales y metaloides tóxicos (MTM). La retención de MTM dentro de los relaves probablemente se produce mediante la formación de carbonatos metálicos de baja solubilidad y por la elevación del $\mathrm{pH}$ que promueve la precipitación de hidróxidos de $\mathrm{Fe}$, los cuales pueden retener $\mathrm{As}$, $\mathrm{Sb}$ y metales.

Aduvire (2006) menciona que la presencia de materiales que contienen sulfuros es condición necesaria para la formación de DAM. Se ha observado que, a mayor contenido de sulfuro mayor es el potencial de formación de aguas ácidas. En cuanto al tamaño de las partículas clasifica para residuos en presas como uniforme al $100 \%$ cuyo tamaño es inferior a 2.0 $\mathrm{mm}$, que reducen la infiltración y la entrada de aire, porque a medida que disminuye el tamaño aumenta la superficie específica.

Considerando los antecedentes expuestos, el objetivo de la presente investigación es determinar si los jales de la mina La Prieta, Chihuahua, México, son generadores de drenaje ácido.

\section{MATERIALES Y MÉTODOS}

\section{Área de estudio}

La zona de estudio de Hidalgo del Parral (Fig. 1) se sitúa sobre roca ígnea del Terciario y rocas sedimentarias del Cretácico y del Neógeno, por ello se observan estos dos tipos de rocas presentes en la zona. Se ubica en la región hidrológica Bravo Conchos (SGM 2019). De acuerdo con Barraza (2015), la presa de jales de la mina La Prieta, tiene una antigüedad aproximada mayor a 300 años, este tiempo se considera a partir de que la mina empezó con la explotación de los minerales y además se supone es la fuente principal de la emisión de los contaminantes al ambiente del área en estudio.

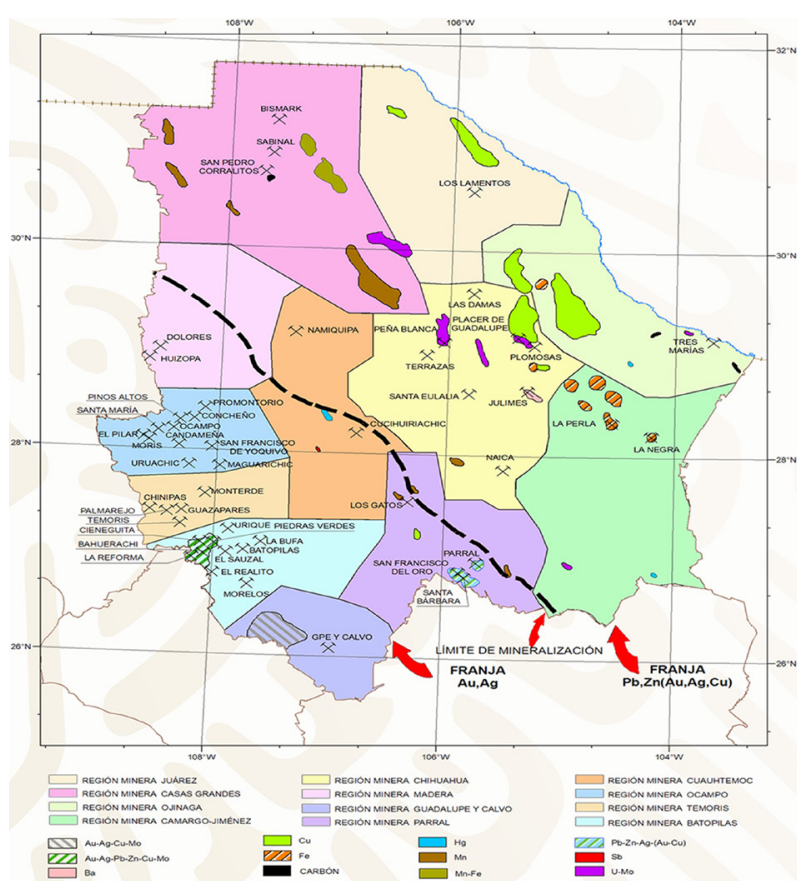

Fig. 1. Ubicación de Hidalgo del Parral en Chihuahua (SGM 2019).

\section{Muestreo y preparación de muestras}

En concordancia con la Norma Mexicana NMXAA-132-SCFI-2006 (SE 2006), Barraza (2015) estableció un plan de muestreo y las especificaciones para la obtención y manejo de 18 muestras. Para ello consideró tres zonas: jales nuevos, jales blancos y jales rojos. El muestreo fue de tipo superficial, con una profundidad de 30 centímetros en cada punto. En cada sitio se recolectaron aproximadamente $500 \mathrm{~g}$ de muestra. Dichas muestras se conservaron en bolsas de plástico con cierre hermético para facilitar su traslado desde Parral, Chihuahua, hasta Ciudad Universitaria en la Ciudad de México. Posteriormente, González (2016) realizó un muestreo complementario en seis puntos, seleccionándolos debido a que Barraza los había caracterizado mediante fluorescencia de rayos X. En la presente investigación se utilizaron las seis muestras recolectadas por González (2016), que habían estado almacenadas durante 2.5 años, aproximadamente, en bolsas de polietileno de baja densidad, herméticamente cerradas y conservadas 
en un ambiente de aproximadamente $4{ }^{\circ} \mathrm{C}$. En la figura 2a, b se muestran los puntos de recolección de ambas investigaciones.
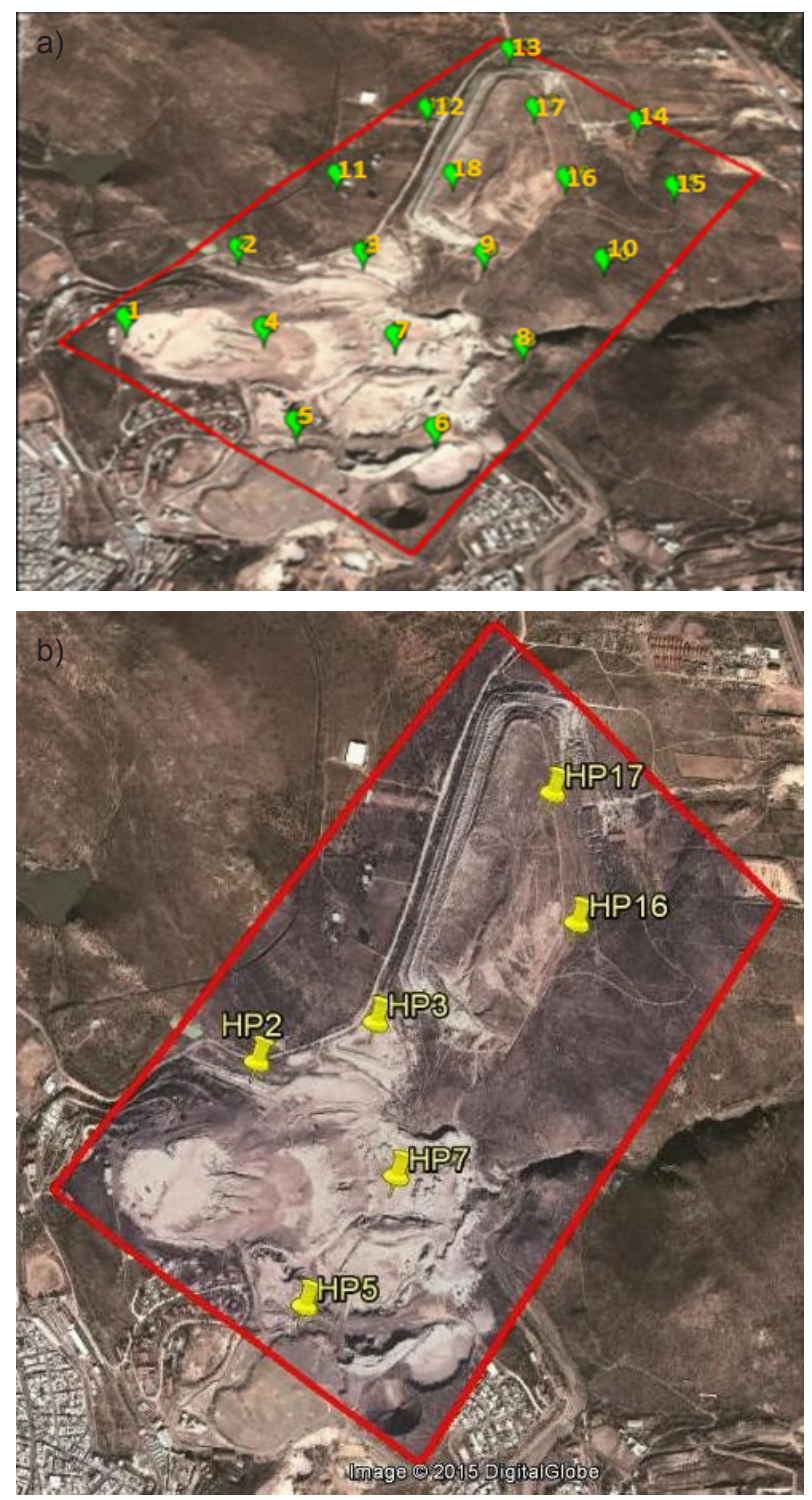

Fig. 2. Ubicación de muestreos de (a) Barraza (2015), (b) Gonzáles (2016). Nota: HP son puntos de muestreo, siglas que corresponden a Hidalgo del Parral.

\section{Métodos y equipos}

Barraza (2015) realizó el análisis mineralógico para las muestras seleccionadas por medio de un difractómetro de geometría Bragg-Brentano PANalytical X'Pert system (monocromador de grafito, rendija automática, radiación $\mathrm{K} \alpha$ del $\mathrm{Cu}$ a $\lambda=1.54061$ $\AA$, potencia de trabajo $45 \mathrm{kV}-40 \mathrm{~mA}$, rango de barrido $4-100^{\circ} 2 \theta$ con paso de $0.017^{\circ} 2 \theta$ y tiempo de medida de $50 \mathrm{~s}$ ). Para la identificación y valoración semicuantitativa de las fases se utilizó el programa XPert Graphics Identify Philips. Por otro lado, la determinación de metales y metaloides presentes en las muestras se llevó a cabo por el método de fluorescencia de rayos $\mathrm{X}$ (XRF) utilizando un espectrómetro secuencial de rayos X Phillips PW 2400.

El potencial de hidrogeno $(\mathrm{pH})$ de las muestras de jales se determinó con base en la Norma Mexicana NMX-AA-013-SCFI-2006 (SE 2012), con ayuda de un potenciómetro Coming 20. El potencial de neutralización y acidez (PN y PA) se obtuvo de acuerdo con lo establecido en la Norma Oficial Mexicana NOM-141-SEMARNAT-2003 (SEMARNAT 2004). Para las pruebas de movilidad se empleó el método "Lixiviación con agua en equilibrio con $\mathrm{CO}_{2}\left(\mathrm{H}_{2} \mathrm{O}\right.$ a $\mathrm{pH}=5.50$ ajustado con burbujeo de $\mathrm{CO}_{2}$ ", establecido en el anexo 5 de la norma mencionada. Las concentraciones de los metales en los lixiviados se determinaron con equipo de absorción atómica marca GBC Avanta.

La determinación de azufre en forma se sulfatos se realizó siguiendo lo estipulado en la NMXAA-74-197 (SECOFI 1981), con ayuda de un equipo Hach 2100 N. La cuantificación del azufre total se llevó a cabo utilizando un equipo de análisis de azufre por infrarrojo Leco S-144DR.

Para obtener los resultados de potencial de neutralización (PN), expresado en $\mathrm{kg}_{\mathrm{CaCO} 3}$ /ton, se utilizó la formula incluida en la NOM-141-SEMARNAT-2003 (SEMARNAT 2004):

$$
P N=\frac{(0.1 \text { x vol en } \mathrm{ml} \mathrm{de} \mathrm{NaOH})}{\text { peso de la muestra }} \times 50
$$

Para calcular el ion sulfato se utilizó la siguiente fórmula (APHA 2003):

$$
\mathrm{mg} \mathrm{SO}_{4}{ }^{2-} / \mathrm{L}=\frac{\mathrm{mg} \mathrm{SO}_{4}{ }^{2-} \times 1000}{\mathrm{~mL} \text { sample }}
$$

Para determinar el porcentaje de sulfuros también se utilizó la fórmula establecida en la NOM-141-SEMARNAT-2003 (SEMARNAT 2004):

$$
\begin{aligned}
& \% \mathrm{~S}^{2-}=\left(\% \mathrm{~S}_{\text {total }}-\% \mathrm{~S}_{\text {sulfatos }}\right) \\
& \mathrm{PA}=\% \mathrm{~S}^{2-} * 31.25
\end{aligned}
$$

\section{RESULTADOS Y DISCUSIÓN}

\section{Caracterización mineralógica}

En el cuadro I se muestra la caracterización mineralógica de las muestras. Como se puede 
CUADRO I. CARACTERIZACIÓN MINERALÓGICA DE LAS MUESTRAS SELECCIONADAS.

\begin{tabular}{|c|c|c|c|c|c|c|c|}
\hline \multirow{2}{*}{ Mineral } & \multirow{2}{*}{$\begin{array}{l}\text { Fórmula } \\
\text { química }\end{array}$} & \multicolumn{6}{|c|}{ Muestras y porcentajes (\%) } \\
\hline & & HP2 & HP3 & HP5 & HP7 & HP16 & HP17 \\
\hline Albita & $\mathrm{NaAlASi}_{3} \mathrm{O}_{8}$ & 5 & 9 & - & 4 & 20 & - \\
\hline Alunita & $\mathrm{KAl}_{3}\left(\mathrm{SO}_{4}\right)_{2}(\mathrm{OH})_{6}$ & 1 & 2 & - & - & 1 & - \\
\hline Barita & $\mathrm{BaSO}_{4}$ & - & 1 & - & 1 & - & - \\
\hline Calcita & $\mathrm{CaCO}_{3}$ & 2 & - & 7 & 1 & 3 & 2 \\
\hline Caolinita & $\mathrm{Al}_{2} \mathrm{Si}_{2} \mathrm{O}_{5}(\mathrm{OH})_{4}$ & 11 & 5 & - & 19 & - & 1 \\
\hline Cuarzo & $\mathrm{SiO}_{2}$ & 43 & 35 & 69 & 52 & 62 & 70 \\
\hline Fluorita & $\mathrm{CaF}_{2}$ & 9 & 7 & 9 & 9 & - & 10 \\
\hline Jarosita & $\mathrm{KFe}_{3}(\mathrm{SO} 4)_{2}(\mathrm{OH})_{6}$ & 1 & - & - & - & - & - \\
\hline Melanderita & $\mathrm{FeSO}_{4} .7 \mathrm{H}_{2} \mathrm{O}$ & 2 & 1 & - & - & - & - \\
\hline Moscovita & $\mathrm{KAl}_{2}\left(\mathrm{AlSi}_{3} \mathrm{O}_{10}\right)(\mathrm{OH})_{2}$ & - & - & - & - & - & - \\
\hline Pirita & $\mathrm{FeS}_{2}$ & 1 & - & 2 & - & - & 1 \\
\hline Plumbojarosita & $\mathrm{Pb}\left(\mathrm{Al}_{3}(\mathrm{OH})_{6}\left(\mathrm{SO}_{4}\right)_{2}\right)_{2}$ & - & 1 & - & 1 & - & - \\
\hline Sericita & $(\mathrm{K}, \mathrm{Na}, \mathrm{Ca}) \mathrm{Al}_{2}(\mathrm{Si}, \mathrm{Al})_{4} \mathrm{O}_{11}$ & 12 & 26 & - & - & - & - \\
\hline Yeso & $\mathrm{CaSO}_{4} \cdot 2 \mathrm{H}_{2} \mathrm{O}$ & 1 & 3 & 6 & 2 & 1 & - \\
\hline
\end{tabular}

Nota: HP son puntos de muestreo, siglas que corresponden a Hidalgo del Parral.

apreciar, los principales minerales que producen drenaje ácido de mina contienen plomo y azufre. El mineral que tiene azufre es la pirita, la cual está presente en muy bajo porcentaje y no se encuentra en todas las muestras. Por otro lado, en los jales se encuentran minerales amortiguadores como los silicatos albita $\left(\mathrm{NaAlSi}_{3} \mathrm{O}_{8}\right)$, caolinita $\left(\mathrm{Al}_{2} \mathrm{Si}_{2} \mathrm{O}_{5}(\mathrm{OH})_{4}\right)$, moscovita $\left(\mathrm{KAl}_{2}\left(\mathrm{AlSi}_{3} \mathrm{O}_{10}\right)(\mathrm{OH})_{2}\right)$ y sericita $(\mathrm{K}, \mathrm{Na}, \mathrm{Ca}) \mathrm{Al}_{2}$ $\left.(\mathrm{Si}, \mathrm{Al})_{4} \mathrm{O}_{11}\right)$; los feldespatos potásicos $\left(\mathrm{KAlSi}_{3} \mathrm{O}_{8}\right)$, y la calcita.

\section{Potencial hidrógeno de las muestras}

Los jales presentan un $\mathrm{pH}$ ligeramente básico. Si a esto se suma el hecho de que la pirita, principal precursor del drenaje ácido, se encuentra sólo en tres muestras y en porcentajes muy bajos (cuadro I), se tiene un primer indicio de que no existe generación de drenaje ácido en los jales de la mina La Prieta. Los valores de $\mathrm{pH}$ en las muestras de jales se observan en la figura 3 .

\section{Determinación del potencial de neutralización}

De acuerdo con la técnica de la NOM-141-SEMARNAT-2003 (SEMARNAT 2004), la determinación del potencial de neutralización (PN) tiene dos mediciones. En primer término, la medición cualitativa de carbonatos: a las muestras de jales se les agregaron tres gotas de $\mathrm{HCl}$ al $25 \%$, lo que comprobó la efervescencia presente en los carbonatos, cuya calificación se aprecia en el cuadro II.

En segundo término, se procedió a medir el poder de neutralización. Con los valores obtenidos de las dos mediciones de los jales mineros en estudio se calculó el PN, el cual varió de 7.5 a $44.0 \mathrm{~kg}$

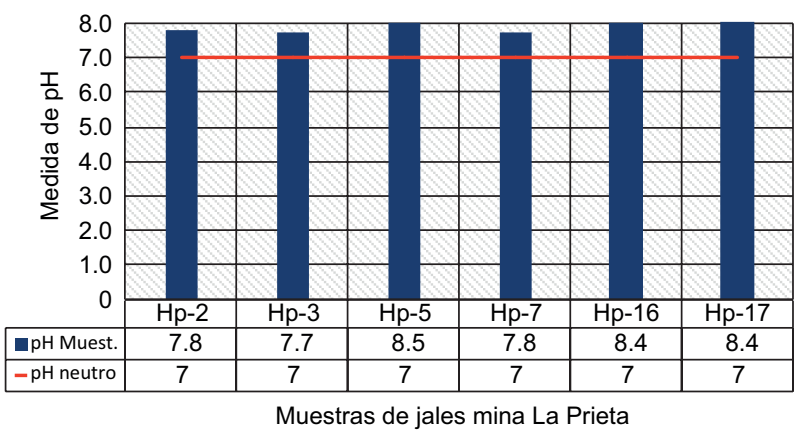

Fig. 3. Resultados de determinación de $\mathrm{pH}$ en las muestras de jales.

CUADRO II. REACCIÓN DE CARBONATOS EN MUESTRAS DE JALES.

\begin{tabular}{cc}
\hline Muestra & Calificativo \\
\hline HP2 & Moderado \\
HP3 & Moderado \\
HP5 & Moderado \\
HP7 & Moderado \\
HP16 & Moderado \\
HP17 & Moderado \\
\hline
\end{tabular}

$\mathrm{CaCO}_{3} / \mathrm{t}$. Esto demuestra que tienen la capacidad de neutralizar la formación de drenaje de ácido de mina (Fig. 4).

\section{Determinación del potencial de acidez}

Para determinar el potencial de acidez (PA) se realizó la extracción de azufre en forma de sulfatos 


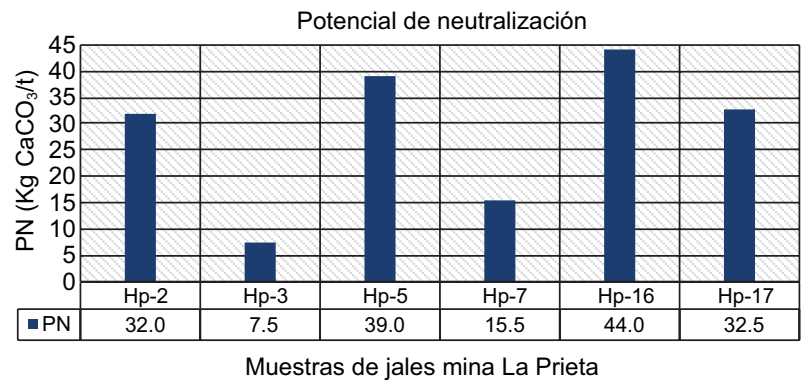

Fig. 4. Resultados de determinación de potencial de neutralización.

con el método turbidimétrico, utilizando un equipo marca Hach $2100 \mathrm{~N}$ (Cuadro III).

CUADRO III. PORCENTAJE DE SULFATOS EN LAS MUESTRAS.

\begin{tabular}{cc}
\hline Muestra & Porcentaje de sulfatos \\
\hline HP2 & 2.086 \\
HP3 & 0.539 \\
HP5 & 0.642 \\
HP7 & 0.205 \\
HP16 & 0.139 \\
HP17 & 0.007 \\
\hline
\end{tabular}

Los sulfatos pueden contribuir a la cuantificación del PA; sin embargo, no se encuentran en todas las muestras (cuadro I) o están en porcentajes muy bajos. Jennings y Dollhopf (2000) corroboraron que los minerales que contienen sulfatos sólo generan acidez si se disuelven. Estos autores autores utilizaron $\mathrm{HCl}(4.91 \mathrm{M})$ y $\mathrm{HNO}_{3}(1.99 \mathrm{M})$ y en sus resultados mencionan que:

- La barita $\left(\mathrm{BaSO}_{4}\right)$ y la jarosita $\left(\mathrm{KFe}_{3}\left(\mathrm{SO}_{4}\right)_{2}(\mathrm{OH})_{6}\right)$ no son extraídos mediante el método Acid-Base Account (ABA) descrito en la NOM-141-SEMARNAT-2003 (SEMARNAT 2004) para la determinación del PA y PN. Además, los autores aseveran que estos minerales pueden causar error al momento de la determinación del PA.

- Para el caso del yeso (CaSO4.2H2O), los autores reportan que este tipo de mineral no tiene asociada generación de acidez.

La determinación de azufre total se efectuó con un equipo de análisis de azufre por infrarrojo Leco S144DR (límite de detección: 1\% en azufre total). Sin embargo, el azufre total no fue detectado. Esto concuerda con los resultados obtenidos en el cuadro I: la pirita se encuentra sólo en tres muestras (HP2, HP5 y HP7) y en porcentajes muy bajos, aunque este mineral es uno de los principales precursores del DAM (Aduvire 2006).

Para el cálculo del PAArmienta (2019) recomienda utilizar la mitad del límite de detección del equipo, es decir, $0.5 \%$ de azufre total. Los resultados del PA se muestran en el cuadro IV.

CUADRO IV. VALORES DEL POTENCIAL DE ACIDEZ (PA) ENCONTRADOS EN LAS MUESTRAS.

\begin{tabular}{lccrr}
\hline Muestra & $\begin{array}{c}\mathrm{S}_{\text {total }} \\
(\%)\end{array}$ & $\begin{array}{c}\text { Sulfatos } \\
(\%)\end{array}$ & \multicolumn{1}{c}{$\begin{array}{c}\mathrm{S}^{2-} \\
(\%)\end{array}$} & PA \\
\hline HP2 & 0.5 & 2.086 & -1.586 & -49.584 \\
HP3 & 0.5 & 0.539 & -0.039 & -1.223 \\
HP5 & 0.5 & 0.642 & -0.142 & -4.455 \\
HP7 & 0.5 & 0.205 & 0.294 & 9.211 \\
HP16 & 0.5 & 0.139 & 0.361 & 11.281 \\
HP17 & 0.5 & 0.007 & 0.492 & 15.405 \\
\hline
\end{tabular}

La relación PN/PA para cada muestra se presentan en la figura 5, donde puede observarse que los valores de las muestras HP2, HP3 y HP5 son menores que el valor de la relación $\mathrm{PN} / \mathrm{PA} \leq 1.2$ por lo que, de acuerdo con la NOM-141-SEMARNAT-2003 (SEMARNAT 2004), son potenciales generadoras de drenaje ácido, mientras que las tres muestras restantes no lo son, ya que el valor de la relación PN/ PA es $>1.2$.

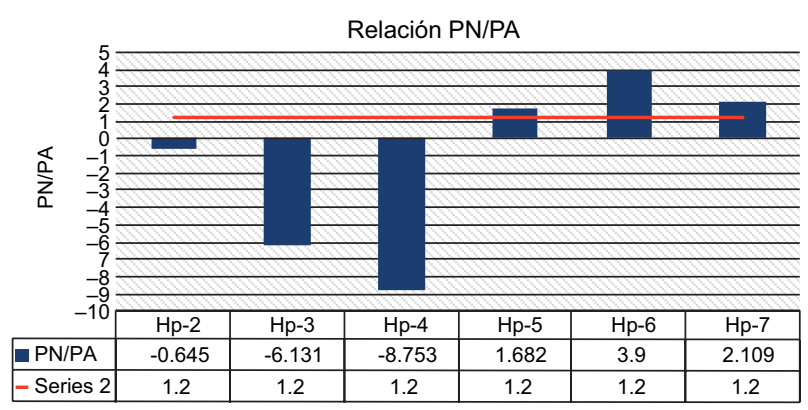

Fig. 5. Relación entre potencial de neutralización y potencial de acidez.

Las muestras provenientes de la presa de jales de la mina La Prieta tienen una antigüedad de más de 300 años (Barraza 2015). Durante este tiempo, el 
horizonte de la presa tuvo varias reacciones químicas y de proceso de lixiviación; sin embargo, aun cuando los carbonatos presentes hubieran reaccionado con el DAM para neutralizarlo, agotándose de manera gradual, los siguientes minerales que actuarían como neutralizantes serían los silicatos y aluminosilicatos (Price 2005). En el cuadro I se muestra la presencia de este tipo de minerales, siendo el cuarzo el que se encuentra en todas las muestras y en cantidades abundantes. Por ello se esperaría que, cuando los carbonatos se agoten con el paso del tiempo, no se genere DAM, ya que estos minerales tienen la capacidad de neutralizar la posible acidez generada.

\section{Concentración de metales y metaloides}

Se determinaron las concentraciones totales (base seca) de metales y metaloides de los elementos potencialmente tóxicos (EPT) en los jales de la mina en estudio, mediante espectrofotometría de absorción atómica y fluorescencia de rayos $\mathrm{X}$ en el caso del antimonio.

En concordancia con la NOM-157-SEMARNAT-2009 (SEMARNAT 2011), los límites máximos permisibles (LMP) son los siguientes: As, $100 \mathrm{ppm}$; $\mathrm{Ba}, 2000$ ppm; Cd, 20 ppm; Pb, 100 ppm, y Sb, 10.6 ppm. Los valores encontrados exceden en demasía los LMP, como se muestra en las figuras 6-10, donde las líneas rojas representan dichos límites.

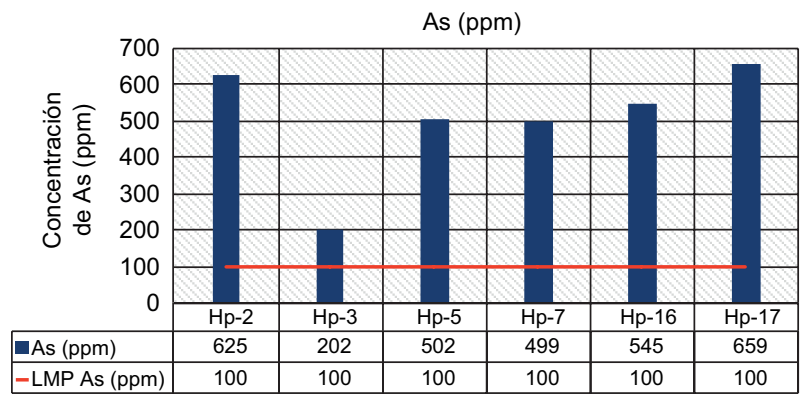

Fig. 6. Concentración de As en las muestras de jales.

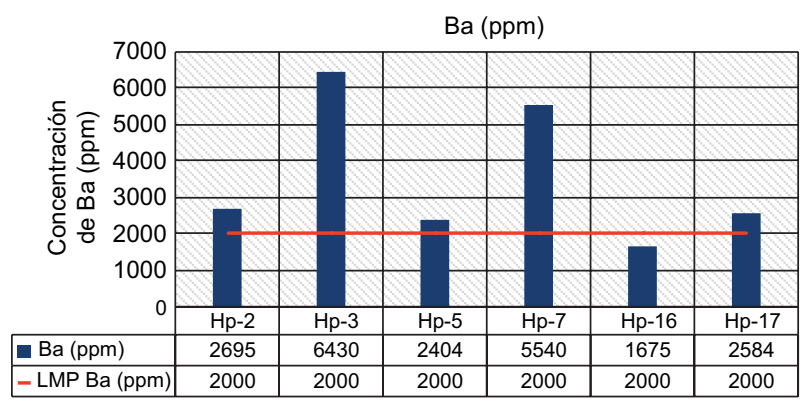

Fig. 7. Concentración de $\mathrm{Ba}$ en las muestras de jales.

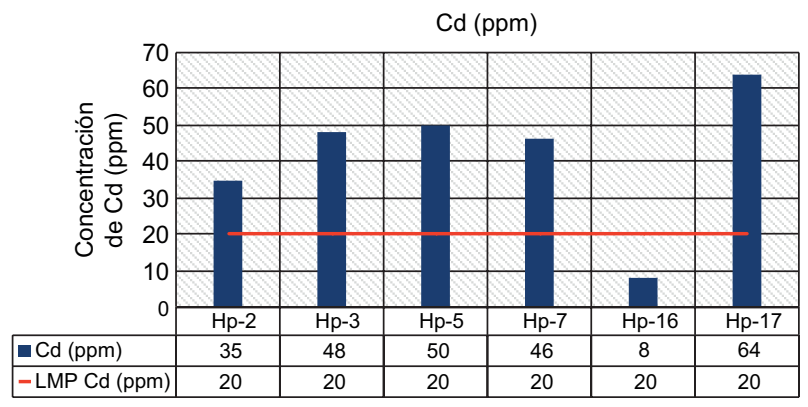

Fig. 8. Concentración de Cd en las muestras de jales.

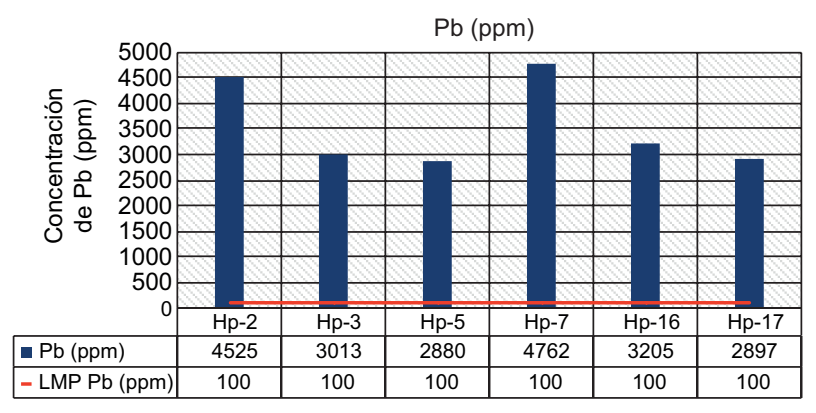

Fig. 9. Concentración de $\mathrm{Pb}$ en las muestras de jales.

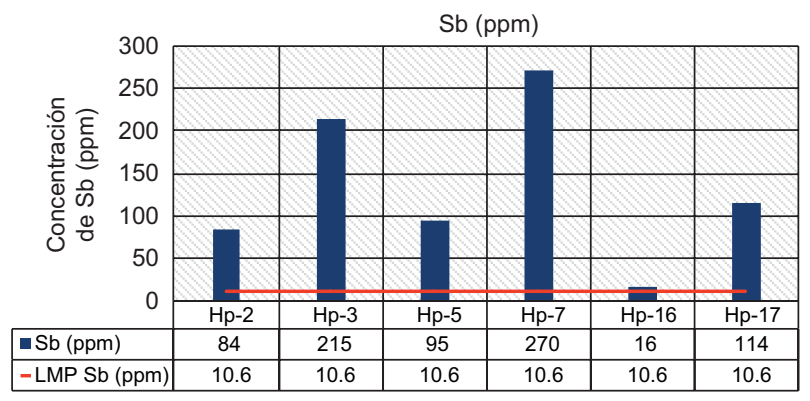

Fig. 10. Concentración de $\mathrm{Sb}$ en las muestras de jales.

Pruebas de movilidad de metales y metaloides

Para las pruebas de movilidad mediante cuarteo se elaboró una muestra compuesta con las muestras HP-2, HP-3, HP-5, HP-7, HP-16 y HP-17 tomadas por González (2016). En el cuadro V se presentan los resultados obtenidos de las pruebas de lixiviación. Para los casos del arsénico, cadmio y plomo se utilizaron los siguientes límites de detección: 3, 0.2 y $2 \mathrm{mg} / \mathrm{L}$, respectivamente.

Los resultados muestran que los metales As, $\mathrm{Cd}$ y $\mathrm{Pb}$ se encuentran por debajo de los límites de detección establecidos y además superan los LMP establecidos en la NOM-157-SEMARNAT-2009 (SEMARNAT 2011); por ello, las muestras de jales seleccionadas de las mina La Prieta no representan un peligro si se toma en cuenta sólo la movilidad de 
CUADRO V. RESULTADOS DE LAS PRUEBAS DE LIXIVIACIÓN.

\begin{tabular}{lcc}
\hline Metal & $\begin{array}{c}\text { Concentración } \\
(\mathrm{mg} / \mathrm{L})\end{array}$ & $\begin{array}{c}\text { LMP } \\
(\mathrm{mg} / \mathrm{L})\end{array}$ \\
\hline Arsénico & $<3$ & 5 \\
Cadmio & $<0.2$ & 1 \\
Plomo & $<2$ & 5 \\
Bario & - & 100 \\
Antimonio & - & 0.53 \\
\hline
\end{tabular}

LMP: límite máximo permisible.

$\mathrm{As}, \mathrm{Cd}$ y $\mathrm{Pb}$. En el caso del bario, Grigorova et al. (2015) mencionan que la barita es la principal fuente de bario en la industria química, por lo que es de esperarse que sea la fuente de bario presente en los jales de la mina La Prieta. Este mineral se encuentra presente en sólo dos muestras (HP3 y HP7) y en bajas cantidades.

Hannor (2000) menciona que la barita tiene una solubilidad extremadamente baja, incluso en condiciones altamente ácidas, además de ser resistente a las condiciones ambientales por la característica mencionada. Con base en lo anterior se esperaría que el Ba no se lixivie ni supere los LMP. En el presente trabajo se utilizó como extractante agua en equilibrio con $\mathrm{CO}_{2}$, con un $\mathrm{pH}$ de 5.5. Por otro lado, Jennings y Dollhopf (2000) utilizaron $\mathrm{HCl}$ y $\mathrm{HNO}_{3}$ como extractantes, encontrando que aun a valores bajos de $\mathrm{pH}$ no ocurre la disolución de la barita y de este modo no es posible la presencia de bario en el lixiviado.

Los jales en estudio provienen de depósitos de mineral tipo skarn, los cuales se caracterizan por ser rocas metamórficas regionales o de contacto constituidas por $\mathrm{Ca}, \mathrm{Mg}$ y $\mathrm{Fe}$, elementos provenientes de un protolito calcáreo (rocas calizas y dolomitas) (Maksaev 2001). Armienta et al. (2012) mencionan que la presencia de calcita y dolomitas en este tipo de yacimientos promueve la retención de $\mathrm{As}, \mathrm{Ba}, \mathrm{Cd}$, $\mathrm{Pb}$ y aumenta la liberación de $\mathrm{Sb}$; sin embargo, los minerales de sulfuro y carbonato controlan el proceso de atenuación natural, evitando así la contaminación ambiental.

\section{Dispersión de los jales}

Barraza (2015) menciona que en la zona de estudio existen dos corrientes de agua (marcadas en color azul, Fig. 11), las cuales son de tipo intermitente, es decir, que sólo en época de lluvias pudieran llegar a tener agua y provocar arrastre de jales.

En el punto donde convergen el río y el arroyo, Barraza (2015) realizó un muestreo (HP8),

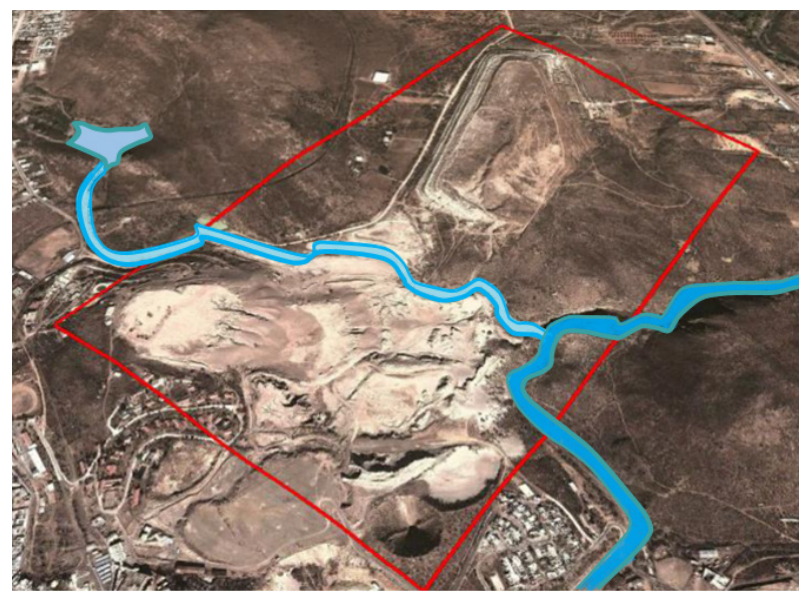

Fig. 11. Corrientes de agua presentes en la zona de estudio (Barraza, 2015).

encontrando bajas concentraciones de EPT (As, 7.42 $\mathrm{mg} / \mathrm{L} ; \mathrm{Pb}, 751.69 \mathrm{mg} / \mathrm{L} ; \mathrm{Ca}, 9.7 \mathrm{mg} / \mathrm{L} ; \mathrm{Ba}, 1324$ $\mathrm{mg} / \mathrm{L}, \mathrm{y} \mathrm{Sb}, 18.2 \mathrm{mg} / \mathrm{L}$ ). Asimismo, asevera que si se toma en cuenta el arrastre producido por el viento no existen barreras para mitigar la dispersión. Los vientos predominantes, de hasta $9.4 \mathrm{~km} / \mathrm{h}$, provienen del suroeste, y la ciudad se encuentra en esa dirección, pro lo que no se descarta que haya arrastre de jales.

\section{CONCLUSIONES}

Las muestras de jales estudiadas de la mina La Prieta, en Hidalgo del Parral, Chihuahua, México, se analizaron para evaluar su capacidad de generación de drenaje ácido. Se concluyó que tres muestras (HP2, HP3 y HP5) son potencialmente generadoras de drenaje ácido, mientras que las restantes no lo son. Por otro lado, metaloides y metales como el As, el $\mathrm{Ca}$ y el $\mathrm{Pb}$ no rebasan los LMP establecidos en la normatividad mexicana. En el caso del Ba y el Sb, la literatura reporta que es poco probable que se lixivien en las condiciones existentes en el sitio de estudio y superen los LMP, debido a la naturaleza de la roca; sin embargo, se requiere efectuar determinaciones analíticas de dichos elementos para establecer si los jales son peligrosos debido a su presencia.

\section{AGRADECIMIENTOS}

El presente trabajo de investigación fue financiado por el Programa de Becas Posdoctorales de la Dirección General de Asuntos del Personal Académico, 
UNAM, y el proyecto PAPIIT con código IN113816. Los autores agradecen a la mina La Prieta por el apoyo brindado para la realización de los muestreos de jales; asimismo, a la Universidad Nacional del Altiplano Puno, Perú (UNA).

\section{REFERENCIAS}

Aduvire O. (2006). Drenaje ácido de mina generación y tratamiento. Dirección de Recursos Minerales y Geoambiente, Instituto Geológico y Minero de España, Madrid, $140 \mathrm{pp}$.

Anawar H.M. (2013). Impact of climate change on acid mine drainage generation and contaminant transport in water ecosystems of semi-arid and arid mining areas. Phys. Chem. Earth, 58-60, 13.21. https://doi. org/10.1016/j.pce.2013.04.002

APHA (2003). Standard methods for the examination of water and wastewater, 4500-S2-F. Iodometric method. 20th ed. American Public Health Association-American Water Works Association-Water Environment Federation, Washington DC.

Armienta M.A., Villaseñor G., Cruz O., Ceniceros N., Aguayo A. y Morton O. (2012). Geochemical processes and mobilization of toxic metals and metalloids in an As-rich base metal waste pile in Zimapán, Central Mexico. Appl. Geochem. 27, 2225-2237. https://doi. org/10.1016/j.apgeochem.2012.01.015

Barraza T.L.A.. (2015). Evaluación de la contaminación del suelo por arsénico, plomo y mercurio en la zona de presa de jales de mina La Prieta en Hidalgo del Parral, Chihuahua, México. Tesis de Maestría. Facultad de Ingeniería, Universidad Nacional Autónoma de México, 106 pp.

Candeias C., Freire Ávila P., Ferreira da Silva E., Ferreira A., Salgueiro A.R. y Teixeira J.P. (2014). Acid mine drainage from the Panasqueira mine and its influence on Zêzere river (central Portugal). J. Afr. Earth Sci. 99 (2), 705-712. https://doi.org/10.1016/j. jafrearsci.2013.10.006

Çelebi E.E. y Öncel M.S. (2016). Determination of acid forming potential of massive sulfide minerals and the tailings situated in lead/zinc mining district of Balya (NW Turkey). J. Afr. Earth Sci. 124, 487-496. https:// doi.org/10.1016/j.jafrearsci.2016.09.014

Chopard A., Benzaazoua M., Bouzahzah H., Plante B. y Marion P. (2017). A contribution to improve the calculation of the acid generating potential of mining wastes. Chemosphere 175, 97-107. https://doi.org/10.1016/j. chemosphere.2017.02.036

Edraki M., Baumgartl T., Manlapig E., Bradshaw D., Franks D.M. y Moran C.J. (2014). Designing mine tailings for better environmental, social and economic outcomes: a review of alternative approaches. J. Clean Prod. 84, 411-420. https://doi.org/10.1016/j. jclepro.2014.04.079

CEHM (2014). Historia minera de Hidalgo del Parral. Centro de Estudios de Historia de México, Fundación Carlos Slim, México [en línea]. http://www.wikimexico.com/articulo/historia-minera-de-hidalgo-del-parral

González S.J.F. (2016). Propuestas para el aprovechamiento de residuos metalúrgicos no ferrosos. México. Tesis de Maestría. Facultad de Ingeniería, Universidad Nacional Autónoma de México, 102 pp.

Grigorova I., Dzhamyarov S. y Nishkov I. (2015). Barite flotation concentrate from Kremikovtzi "black" tailings. Journal of International Scientific Publications: Materials, Methods \& Technologies 9, 561-577.

Hannor J.S. (2000). Barite-celestine geochemistry and environments of formation. Revi. Mineral. Geochem. 40 (1), 193-275. https://doi.org/10.2138/rmg.2000.40.4

Jennings S.R. y Dollhopf D.J. (1995). Acid-base account effectiveness for determination of mine waste potential acidity. J. Hazard. Mat. 41, 161-175. https://doi. org/10.1016/0304-3894(95)00003-D

Johnson D.B. y Hallberg K.B. (2005). Acid mine drainage remediation options: A review. Sci. Total Environ. 338, 3-14. https://doi.org/10.1016/j.scitotenv.2004.09.002

Khademi H., Abbaspour A., Martínez-Martínez S., Gabarrón M., Shahrokh V., Faz A. y Acosta J.A. (2018). Provenance and environmental risk of windblown materials from mine tailing ponds, Murcia, Spain. Environ. Pollut. 241, 432-440. https://doi.org/1016/j. envpol.2018.05.084

Lèbre E., Corder G.D. y Golev A. (2017). Sustainable practices in the management of mining waste: A focus on the mineral resource. Miner. Eng. 107, 34-42. https:// doi.org/10.1016/j.mineng.2016.12.004

Lindsay M.B.J., Moncur M.C., Bain J.G., Jambor J.L., Ptacek C.J. y Blowes D.W. (2015). Geochemical and mineralogical aspects of sulfide mine tailings. Appl. Geochem. 57, 157-177. https://doi.org/10.1016/j. apgeochem.2015.01.009

Maksaev V. (2001). Metalogénesis [en línea]. http://www. medellin.unal.edu.co/ rrodriguez/victor-maksaev/ index-metalo.htm

Moodley I., Sheridan C.M., Kappelmeyer U. y Akcil A. (2018). Environmentally sustainable acid mine drainage remediation: Research developments with a focus on waste/by-products. Miner. Eng. 126, 207-220. https://doi.org/10.1016/j.mineng.2017.08.008

Nordstrom D.K., Blowes D.W. y Ptacek C.J. (2015). Hydrogeochemistry and microbiology of mine drainage: An update. Appl. Geochem. 57, 3 -16. https://doi. org/10.1016/j.apgeochem.2015.02.008 
NMX-AA-74 (1981) Análisis de agua - determinación del ion sulfato. Norma Mexicana. Secretaria de Comercio y Fomento Industrial. México.

Pabst T., Bussière B., Aubertin M. y Molson J. (2018). Comparative performance of cover systems to prevent acid mine drainage from pre-oxidized tailings: A numerical hydro-geochemical assessment. J. Contam. Hydrol. 214, 39-53. https://doi.org/10.1016/j.jconhyd.2018.05.006

Plaza F., Wen Y. y Liang X. (2018). Acid rock drainage passive remediation using alkaline clay: Hydro-geochemical study and impacts of vegetation and sand on remediation. Sci. Total Environ. 637-638, 1262-1278. https://doi.org/10.1016/j.scitotenv.2018.05.014

Price W.A. (2005). Review of 2004 waste rock Storage Area Seepage and Waste Rock Survey Report for Ekati Mine. Independent Environmental Monitoring Agency, Canadá, 34 pp.

Sánchez-Andrea I, Sanz J.L., Bijmans M.F.M. y Stams A.J.M. (2014). Sulfate reduction at low $\mathrm{pH}$ to remediate acid mine drainage. J. Hazard. Mater. 269, 98-109. https://doi.org/10.1016/j.jhazmat.2013.12.032

SE (2006). Norma Mexicana NMX-AA-132-SCFI-2006. Muestreo de suelos para la identificación y la cuantificación de metales y metaloides, y manejo de la muestra. Secretaría de Economía. Diario Oficial de la Federación, México, 6 de marzo.

SE (2012). Norma Mexicana NMX-AA-013-SCFI-2006. Residuos sólidos - determinacón de $\mathrm{pH}$ - Método de prueba. Secretaría de Economía. Diario Oficial de la Federación, México, 6 de diciembre.

SE (2020). Manual del Inversionista en el Sector Minero Mexicano. Secretaria de Economía. México. [en línea] https://www.economia.gob.mx/files/gobmx/mineria/ manual_del_inversionista.pdf
SECOFI (1981). NMX-AA-74. Análisis de agua - determinación del ion sulfato. Norma Mexicana. Secretaria de Comercio y Fomento Industrial. México.

SEMARNAT (2004). Norma Oficial Mexicana NOM141-SEMARNAT. Que establece el procedimiento para caracterizar los jales, así como las especificaciones y criterios para la caracterización y preparación del sitio, proyecto, construcción, operación y postoperación de presas de jales. Secretaría de Medio Ambiente y Recursos Naturales. Diario Oficial Diario Oficial de la Federación, México, 13 de septiembre.

SEMARNAT (2011). Norma Oficial Mexicana NOM157-SEMARNAT-2009. Que establece los elementos y procedimientos para instrumentar planes de manejo de residuos mineros. Secretaria de Medio Ambiente y Recursos Naturales Diario Oficial Diario Oficial de la Federación, México, 30 de agosto.

SGM (2019). Panorama minero del estado de Chihuahua. Servicio Geológico Mexicano, Secretaría de Economía, México, 14 pp

Sheoran A.S. y Sheoran V. (2006). Heavy metal removal mechanism of acid mine drainage in wetlands: A critical review. Miner. Eng. 19, 105-116. https://doi. org/10.1016/j.mineng.2005.08.006

Yang W.-J., Ding K.-B., Zhang P., Qiu H., Cloquet C., Wen H.-J., Morel J.-L., Qiu R.-L. y Tang Y.-T. (2019). Cadmium stable isotope variation in a mountain area impacted by acid mine drainage. Sci. Total Environ. 646, 696-703. https://doi.org/10.1016/j.scitotenv.2018.07.210

Zhou J., Nyirenda M.T., Xie L., Li Y., Zhou B., Zhu Y. y Liu H. (2017). Mine waste acidic potential and distribution of antimony and arsenic in waters of the Xikuangshan mine, China. Appl. Geochem. 77, 5261. https://doi.org/10.1016/j.apgeochem.2016.04.010 